\title{
Evaluation des Effets Fertilisants et Embryo-Toxiques de L'extrait Aqueux des Feuilles de Cnestis ferruginea Chez le Rat
}

\author{
Zougrou N'guessan Ernest, \\ Tovi Wahon Marie-Odile, \\ Blahi Adélaïde Nadia Méa, \\ Kouakou Koffi,
}

Laboratoire d'Endocrinologie et Biologie de la Reproduction, UFR Biosciences, Université Félix Houphouët-Boigny, Côte d'Ivoire

Doi:10.19044/esj.2019.v15n27p231 URL:http://dx.doi.org/10.19044/esj.2019.v15n27p231

\section{Résumé}

L'objectif de ce travail est d'évaluer les effets fertilisants et embryotoxiques de l'extrait aqueux de Cnestis ferruginea chez le rat. D'abord, 3 lots de rattes (témoin, traitées EACF50 et EACF100) ont été accouplées avec les mâles non traités. Ensuite, 3 lots de rats mâles (témoin, traités EACF50 et EACF100) ont été accouplés avec les femelles non traitées. Enfin, deux groupes de rats, I (mâles) et II (femelles) ont été subdivisés en trois lots chacun (témoin, traités EACF50 et EACF100) et ont été accouplés en fonction de la dose. Les résultats ont montré $100 \%$ de gestation chez les femelles traitées, mais une augmentation du taux d'avortement post-implantation avec l'EACF100. Concernant le test de mâles traités, le taux de gestation, copulation et fertilité étaient de $75 \%, 83,33 \%$ et $100 \%$ respectivement pour les témoins, EACF50 et EACF100. Les taux d'avortement pré- et postimplantation ont baissés avec les deux doses. Le test de mâles traités accouplés avec les femelles traitées a montré un taux de gestation, un index de copulation et un index de fertilité de $100 \%$ par rapport aux témoins $(83,33 \%)$. Concernant les poids de l'utérus, du fœtus et du placenta, l'extrait a induit une augmentation significative chez les femelles traitées. Les études histologiques de l'ovaire ont montré une augmentation significative du diamètre des corps jaunes $(35,14 \%)$ et du diamètre des glandes utérines de 16,05\% (EACF50) et 18,39\% (EACF100). En conclusion, Cnestis ferruginea améliore la fertilité chez les mâles et les femelles. Mais, ces effets positifs peuvent être entravés par les fortes doses. 
Mots-clés: Fertilisant, Embryo-toxique, Cnestis ferruginea, Gestation, Avortement

\title{
Evaluation of Fertilizing and Embryo-toxic Effects of Aqueous Extract of Cnestis Ferruginea Leaves in Rats
}

\author{
Zougrou N'guessan Ernest, \\ Tovi Wahon Marie-Odile, \\ Blahi Adélaïde Nadia Méa, \\ Kouakou Koffi,
}

Laboratoire d'Endocrinologie et Biologie de la Reproduction, UFR Biosciences, Université Félix Houphouët-Boigny, Côte d'Ivoire

\begin{abstract}
The objective of this work is to evaluate the fertilization and embryotoxic effects of aqueous extract of Cnestis ferruginea in rats. First, 3 batches of rats (control, treated $\mathrm{AECF}_{50}$ and $\mathrm{AECF}_{100}$ ) were mated with untreated males. Then, 3 batches of male rats (control, treated $\mathrm{AECF}_{50}$ and $\mathrm{AECF}_{100}$ ) were mated with untreated females. Finally, two groups of rats, I (males) and II (females) were subdivided into three batches each (control, treated $\mathrm{AECF}_{50}$ and $\mathrm{AECF}_{100}$ ) and were mated according to the dose. The results showed $100 \%$ gestation in the treated females, but an increase in the post-implantation abortion rate with the $\mathrm{AECF}_{100}$. Regarding the test of treated males, the pregnancy, copulation and fertility rates were $75 \%, 83.33 \%$ and $100 \%$ for controls, $\mathrm{AECF}_{50}$ and $\mathrm{AECF}_{100}$, respectively. Pre- and post-implantation abortion rates decreased with both doses. The test of treated males mated with the treated females showed a pregnancy rate, a copulation index, and a $100 \%$ fertility index compared to controls $(83.33 \%)$. For the uterine, fetal and placental weights, the extract induced a significant increase in the treated females. Histological studies of the ovary showed a significant increase in the diameter of the corpus luteum $(35.14 \%)$ and the diameter of the uterine glands by $16.05 \%\left(\mathrm{AECF}_{50}\right)$ and $18.39 \%\left(\mathrm{AECF}_{100}\right)$. In conclusion, Cnestis ferruginea improves fertility in males and females. But, these positive effects can be hindered by high doses.
\end{abstract}

Keywords: Fertilization, Embryo-toxic, Cnestis ferruginea, Pregnancy, Abortion 


\section{Introduction}

Cnestis ferruginea Vahl ex DC, une espèce de la famille des Connaraceae, a une large répartition en Afrique de l'Ouest. En effet, cette plante est rencontrée depuis le Sénégal jusqu'à l'Ouest du Cameroun ainsi que dans d'autres parties de l'Afrique tropicale (Burkill, 1985). C'est un arbuste sarmenteux lianescent pouvant avoir les vrilles ligneuses (Berhaut, 1967). Elle est pérenne de 3,0 à 3,6 m de hauteur (Irvine, 1961), pouvant atteindre $6 \mathrm{~m}$ (Berhaut, 1954; Burkill, 1985). Cnestis ferruginea, au Nigéria chez les Yorouba, est appelée gboyingboyin ou omaaja, en Côte d'Ivoire, chez les baoulés, elle est nommée wonsienblakassa (Burkill, 1985 ; Irvine, 1961).

Les études ethnobotaniques ont montré qu'en Côte d'Ivoire, la sève des feuilles ou au Sénégal, le jus de fruit de Cnestis ferruginea instillé sur l'œil est un remède contre les troubles occulaires tels que la conjonctivite (Kerharo, 1974 ; Kerharo et Adam, 1974 ; Okafor et Ham, 1999). La pulpe du fruit est utilisée pour nettoyer les dents et remédier aux infections des gencives (Irvine, 1961 ; Gill, 1992 ; Okafor et Ham, 1999). Dans la santé de la reproduction, en Afrique de l'Ouest, les feuilles sont considérées comme abortives et les racines sont utilisées dans le traitement des troubles ovariens et les dysménorrhées chez la femme. Ces racines sont utilisées comme aphrodisiaque chez les hommes. Précisément, en Côte d'Ivoire et au Burkina Faso, les racines sont utilisées, par lavement, pour traiter les troubles gynécologiques et l'écoulement urétral (Gill, 1992 ; Okafor et Ham, 1999). En outre, en Côte d'Ivoire, les feuilles sont utilisées pour traiter la ménorragie (N'guessan et al., 2006).

Les études phytochimiques réalisées sur cette plante ont montré qu'elle contient la octacosanylstearate et la 1-myristo-2-stearo-3-palmitine, l'isoflavone glycoside ; l'afrormosin-7-o-beta-D-galactoside (Ogbechie et al., 1987 ; Parvez et Rahman, 1992). D'autres composés comme le squalène, l'alcool myricyl, la cyamidine, la delphimidine et l'apigenidine ont été isolées de la plante (Ogbede et al., 1986). L'extrait aqueux des racines de $C$. ferruginea contient les alcaloïdes, les flavonoïdes, les saponosides, les anthroquinines et les tanins (Yakubu et al., 2011).

Les études pharmacologiques réalisées sur $C$. ferruginea ont montré que cette plante possède des activités anti-bactériennes et anti-oxydantes (Akharaiyi et al., 2012), anti convulsivantes (Déclume et al., 1984), anti-stress (Ishola et al., 2007 ; Yakubu et al., 2011), laxatives (Yakubu et al., 2011), Hypoglycémiantes (Adisa et al., 2010) et analgésiques et anti inflammatoires (Ishola et al., 2011). Dans le domaine de la reproduction, l'extrait $C$. ferruginea restaure la compétence sexuelle chez le rat mâle (Yakubu et Nurudeen, 2012) et possède des effets œstrogéniques chez la ratte (Zougrou et al., 2016). 
Le but de ce travail a été d'évaluer les effets fertilisants et embryo-toxiques de l'extrait aqueux de Cnestis ferruginea chez le rat.

\section{Matériel et Méthodes}

\section{Matériel vegetal}

Les feuilles fraîches de Cnestis ferruginea ont été récoltées en novembre dans la région de la Nawa, département de Soubré, sous-préfecture de Buyo, précisément dans le village appelé Trawlinkro (V8) (Côte d'Ivoire) (figure 1). Un échantillon de cette plante a été identifié et authentifié par le Professeur Aké-Assi au Laboratoire de Botanique et Biologie végétale de l'Université Félix Houphouët-Boigny sur la base de caractères taxonomiques et par comparaison directe avec les spécimens d'herbier $N^{\circ} 3974,4327$ et 15116 disponible au Centre national de la floriculture (UJC).

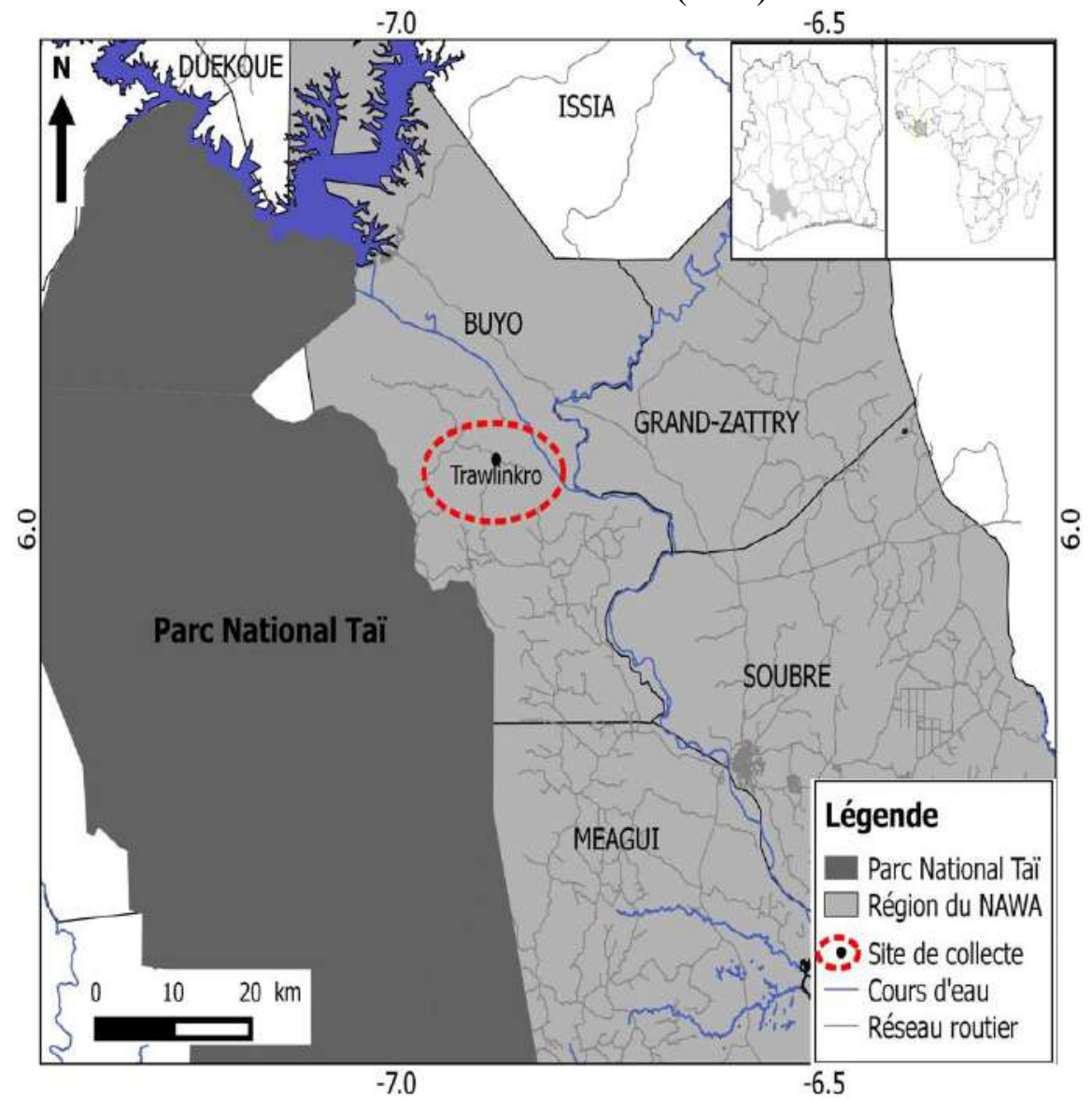

Figure 1 : Localisation de la zone de récolte des feuilles de Cnestis ferruginea dans la région du Nawa (Côte d'Ivoire) 


\section{Préparation de l'extrait}

Les feuilles de C. ferruginea récoltées ont été rincées à l'eau, séchées à l'ombre (à l'abri du soleil), à la température du laboratoire (ambiante). Les feuilles séchées ont été moulues à l'aide d'un moulin de marque IKA A10 Labortechnik (Allemagne) de manière à obtenir une poudre. La poudre obtenue a été portée à macération en mélangeant $50 \mathrm{~g}$ et $1,5 \mathrm{~L}$ d'eau distillée et agitée pendant 24 heures par un agitateur magnétique (JANKE \& KUNTELIKA Labortechnik, Allemagne). Après filtration trois fois sur un tissu propre, trois fois sur du coton hydrophile et trois fois sur du papier filtre Whatman $\mathrm{n}^{\circ} 1$, le filtrat a été placé dans une étuve de marque Memmert à $50^{\circ} \mathrm{C}$ jusqu'à obtenir un extrait sec sous forme de paillette.

\section{Matériel animal}

Les rats mâles et femelles adultes (Rattus norvegicus, Muridae), de souche Wistar, pesant entre 140-160 g et âgés de 55 à 65 jours, proviennent du vivarium de l'Ecole Normale Supérieure (ENS). Ces rats ont servi à la réalisation de l'étude de la fertilité de l'extrait aqueux de $C$. ferruginea.

\section{Méthodes expérimentales}

\section{Fertilité et embryo-toxicité chez la femelle}

24 rattes vierges sont reparties en 3 lots de 8 animaux chacun et traitées comme suit :

- Lot 1 (témoin), eau distillée ;

- Lot $2\left(\mathrm{EACF}_{50}\right), 50 \mathrm{mg} / \mathrm{kg}$ de $\mathrm{PC}$ d'extrait aqueux de C. ferruginea ;

- Lot $3\left(\mathrm{EACF}_{100}\right), 100 \mathrm{mg} / \mathrm{kg}$ de $\mathrm{PC}$ d'extrait aqueux de C. ferruginea.

Ces animaux ont reçu par voie orale $1 \mathrm{~mL}$ soit d'eau distillée (témoins), soit d'extrait aqueux de C. ferruginea pendant 30 jours. Au $20^{\text {ème }}$ jour de traitement, les rattes de chaque lot ont été réparties en 4 sous lots de 2 femelles. Dans chaque sous lot est introduit un rat mâle (ayant prouvé sa fertilité auparavant) pendant les 10 derniers jours de traitement.

\section{Fertilité et embrio-toxicité chez le mâle}

18 rats mâles sont divisés en trois lots de 6 sujets chacun et traités comme suit :

- Lot 1 (témoin), eau distillée ;

- Lot $2\left(\mathrm{EACF}_{50}\right), 50 \mathrm{mg} / \mathrm{kg}$ de $\mathrm{PC}$ d'extrait aqueux de C. ferruginea ;

- Lot $3\left(\mathrm{EACF}_{100}\right), 100 \mathrm{mg} / \mathrm{kg}$ de PC d'extrait aqueux de C. ferruginea.

Ces animaux ont reçu par voie orale $1 \mathrm{~mL}$ soit d'eau distillée, soit d'extrait aqueux de $C$. ferruginea pendant 60 jours. Au 50 ème jour de traitement, chaque rat est placé en présence de deux femelles (ayant prouvé leur fertilité auparavant) pendant les 10 jours de traitement restant. 


\section{Fertilité et embrio-toxicité chez le mâle et la femelle}

Pour ce test, 54 rats sont repartis en deux groupes. Le groupe I est constitué de 18 mâles divisés en trois lots de 6 sujets chacun et traités comme suit :

- Lot 1 (témoin), eau distillée ;

- $\quad$ Lot $2\left(\mathrm{EACF}_{50}\right), 50 \mathrm{mg} / \mathrm{kg}$ de $\mathrm{PC}$ d'extrait aqueux de C. ferruginea ;

- Lot $3\left(\mathrm{EACF}_{100}\right), 100 \mathrm{mg} / \mathrm{kg}$ de PC d'extrait aqueux de C. ferruginea.

Le groupe II est constitué de 36 femelles divisées en trois lots de 12 sujets chacun comme suit :

- Lot 1 (témoin), eau distillée ;

- $\quad$ Lot $2\left(\mathrm{EACF}_{50}\right), 50 \mathrm{mg} / \mathrm{kg}$ de PC d'extrait aqueux de C. ferruginea ;

- Lot 3 (EACF $\left.\mathrm{E}_{100}\right), 100 \mathrm{mg} / \mathrm{kg}$ de PC d'extrait aqueux de C. ferruginea.

Ces rats recevaient quotidiennement $1 \mathrm{~mL}$ soit d'eau distillée, soit d'extrait aqueux de $C$. ferruginea pendant 60 jours pour le groupe I et 30 jours pour le groupe II.

$\mathrm{Au} 50^{\text {ème }}$ jour de traitement pour le groupe I (mâle) et $20^{\text {ème }}$ jour de traitement pour le groupe II (femelle), ces animaux sont mis en accouplement pendant les 10 jours restant en fonction de la dose. C'est-à-dire les témoins sont croisés entre eux et les traités de chaque dose entre eux avec un ratio de 1 mâle pour deux femelles.

\section{Détection de gestation et poids corporel}

Tous les matins entre 7 et 8 heures, les frottis vaginaux ont été réalisés sur chaque ratte après l'introduction du mâle. Ainsi, une solution saline $9 \%$ (v/v) d'environ $10 \mu \mathrm{L}$ a été soigneusement introduite dans le vagin à l'aide d'une micropipette puis prélevée et observée au microscope photonique (Hafez, 1970). La présence de spermatozoïdes dans le flux vaginal d'une ratte est synonyme de gestation et ce jour est considéré comme le jour 1 de gestation. Cette ratte a été pesée et retirée de la cage d'accouplement. Cependant elle continue d'être traitée jusqu'au 30 ème jour (pour les rattes traitées). Après les 10 jours d'accouplement (environ deux cycles œstraux), les femelles gestantes ont été enregistrées. Au $15^{\text {ème }}$ jour de gestation, les rattes ont été pesées, sacrifiées après anesthésie à l'éther et autopsiées.

\section{Paramètres de fertilités et poids des organes}

Au cours de l'autopsie, le nombre de site d'implantation, le nombre de site de résorption et le nombre de fœtus viable ont été comptés et enregistrés. L'utérus contenant les fœtus et les ovaires ont été disséqués et pesés. Chaque fœtus et son placenta ont été libérés de l'utérus et pesés séparément. 


\section{Etude histologique}

Un fragment de l'utérus et les ovaires de chaque ratte ont été immédiatement fixés dans du formol 10\%. Après les bains d'alcool à degré croissant et de toluène, les organes ont été inclus dans de la paraffine puis coupés à l'aide d'un microtome à une épaisseur de $5 \mu \mathrm{m}$. les coupes ont été colorées par la technique d'hématoxyline-éosine.

\section{Analyses statistiques}

Les analyses des données et les représentations graphiques ont été réalisées en utilisant le logiciel GraphPad Prism 5.01 (Microsoft, USA). Les valeurs sont présentées sous forme de moyenne \pm SEM et les données ont été évaluées par la méthode d'analyse d'ANOVA one-way suivi du test de comparaison multiple de Tukey au seuil de 5\%. Si $\mathrm{P}<0,05$ la différence entre les valeurs est considérée comme significative, si $\mathrm{P}<0,01$ cette différence est considérée comme très significative, si $\mathrm{P}<0,001$ elle est considérée comme hautement significative et si $\mathrm{P}>0,05$ cette différence n'est pas significative.

\section{Résultats}

\section{Effet de Cnestis ferruginea sur la fertilité de la ratte} Effets sur le poids corporel, le taux de gestation et quelques paramètres de la fertilité

En début de gestation, il n'y avait aucune différence significative entre le poids des rattes des différents lots. Après 15 jours de gestation, le poids corporel des rattes prétraitées avaient augmenté significativement par rapport aux témoins. En effet, le poids corporel des rattes prétraitées avec l'EACF 50 a augmenté de $8,61 \%(\mathrm{p}<0,05)$ tandis que celui des rattes prétraités avec la dose d'EACF 100 s'est accru de 17,68\% (p<0,001).

$\mathrm{Au}$ regard des paramètres de fertilité, les rattes ayant reçu la dose $\mathrm{EACF}_{50}$ et $\mathrm{EACF}_{100}$ ont présenté un taux de gestation de $100 \%$ soit $8 / 8$ respectivement au $3^{\text {ème }}$ et au $2^{\text {ème }}$ jour d'accouplement. Le lot témoin n'a présenté que $87,50 \%$ soit $7 / 8$ jusqu'au dernier jour d'accouplement $(10$ ème jour).

Concernant l'index de copulation, l'index de fécondité et l'index de fertilité, les rattes traitées avec les différentes doses ont présenté un taux de $100 \%$ dans chaque cas. Ces taux étaient de $87,5 \%, 100 \%$ et $87,5 \%$ respectivement pour l'index de copulation, l'index de fécondité et l'index de fertilité en ce qui concerne les témoins (Tableau I). 


\section{Effets de Cnestis ferruginea sur quelques paramètres de la fertilité après autopsie}

Les rattes gestantes issues du traitement avec l'EACF ont présenté une augmentation du nombre de corps jaune de façon dose dépendante. Mais, cette augmentation n'est pas statistiquement significative comparée aux témoins.

$\mathrm{Au}$ niveau des sites d'implantation, leur nombre s'est accru significativement avec les deux traitements comparativement aux témoins. Ainsi, on assiste à une augmentation de 29,82\% ( $p<0,05)$ et de $61,40 \%$ $(\mathrm{p}<0,01)$ respectivement pour les rattes traitées avec l'EACF 50 et l'EACF 100 .

Le dénombrement des sites de résorption chez les rattes traitées est significatif $(p<0,05)$ seulement avec $1^{\prime} E A C F_{100}$. Ainsi, cette dose a produit une augmentation de $600 \%$ de site de résorption par rapport aux témoins. La baisse du nombre de site de résorption avec l'EACF 50 par rapport au témoin n'est pas significative.

En ce qui concerne le nombre total de fœtus, l'extrait a provoqué une augmentation très significative $(p<0,01)$ de ce paramètre avec l'EACF 50 . En effet, cette augmentation est de $29,54 \%$ par rapport aux témoins.

Dans cette expérience, les résultats montrent que tous les fotus observés étaient viables et que, l'extrait a provoqué une augmentation très significative $(\mathrm{p}<0,01)$ de leur nombre avec les deux traitements par rapport au témoin. Ainsi, l'augmentation du nombre de fœtus viable était de $32,73 \%$ et de $41,82 \%$ respectivement avec l'EACF 50 et l'EACF 100 .

La détermination du pourcentage d'avortement pré-implantation a montré une diminution de façon dose dépendante par rapport aux témoins. Pendant que le taux d'avortement post-implantation a diminué pour le traitement utilisant l'EACF 50 (1,35\%), l'EACF 100 a augmenté ce taux de $15,22 \%$ par rapport aux témoins (Tableau II). 
Tableau I : Effets de Cnestis ferruginea sur le poids corporel, le taux de gestation et quelques paramètres de la fertilité des rattes.

\begin{tabular}{ccccccc}
\hline Groupe & Poids initial (g) & Poids finale $(\mathbf{g})$ & $\begin{array}{c}\text { Nombre de } \\
\text { femelles } \\
\text { gestantes }\end{array}$ & $\begin{array}{c}\text { Index de } \\
\text { copulation (\%) }\end{array}$ & $\begin{array}{c}\text { Index de } \\
\text { fécondité }(\%)\end{array}$ & $\begin{array}{c}\text { Index de } \\
\text { fertilité }(\%)\end{array}$ \\
\hline Témoin & $151,4 \pm 2,214$ & $175,3 \pm 3,337$ & $7 / 8$ & 87,500 & 100,000 & 87,500 \\
EACF $_{\mathbf{5 0}}$ & $149,2 \pm 4,420$ & $190,4 \pm 5,487^{*}$ & $8 / 8$ & 100,000 & 100,000 & 100,000 \\
EACF $_{\mathbf{1 0 0}}$ & $152,0 \pm 4,214$ & $206,3 \pm 0,4592^{* * *}$ & $8 / 8$ & 100,000 & 100,000 & 100,000 \\
\hline
\end{tabular}

Les valeurs sont des moyennes $\pm \mathrm{ESM}(\mathrm{n}=12 / \mathrm{lots}) .{ }^{*} \mathrm{p}<0.05$, *** $\mathrm{p}<0,001$. EACF $_{50}:$ Extrait Aqueux de C. ferruginea $(50 \mathrm{mg} / \mathrm{kg}$ de P.C) EACF 100 : Extrait Aqueux de C. ferruginea $(100 \mathrm{mg} / \mathrm{kg}$ de P.C)

Tableau II : Effets de Cnestis ferruginea sur quelques paramètres de la fertilité après autopsie des rattes

\begin{tabular}{ccccccc}
\hline Groupe & $\begin{array}{c}\text { Nb de corps } \\
\text { jaune }\end{array}$ & $\begin{array}{c}\text { Nb de site } \\
\text { d'implantation }\end{array}$ & $\begin{array}{c}\text { Nb de site de } \\
\text { résorption }\end{array}$ & $\begin{array}{c}\text { Nb de foetus } \\
\text { viable }\end{array}$ & $\begin{array}{c}\text { Avortement pré- } \\
\text { implantation } \\
(\boldsymbol{\%})\end{array}$ & $\begin{array}{c}\text { Avortement } \\
\text { post- } \\
\text { implantation } \\
(\boldsymbol{\%})\end{array}$ \\
\hline Témoin & $11,130 \pm 0,680$ & $7,125 \pm 0,743$ & $0,250 \pm 0,164$ & $6,875 \pm 0,289$ & 35,98 & 3,51 \\
EACF $_{\mathbf{5 0}}$ & $12,250 \pm 0,818$ & $9,250 \pm 0,940^{*}$ & $0,125 \pm 0,125$ & $9,125 \pm 0,315^{* *}$ & 24,49 & 1,35 \\
EACF $_{\mathbf{1 0 0}}$ & $13,000 \pm 0,964$ & $11,500 \pm 0,802^{* *}$ & $1,750 \pm 0,267^{*}$ & $9,750 \pm 0,288^{* *}$ & 11,54 & 15,22 \\
\hline
\end{tabular}

Les valeurs sont des moyennes \pm ESM $(\mathrm{n}=12 / \mathrm{lots}) .{ }^{*} \mathrm{p}<0.05$, ${ }^{*} \mathrm{p} \mathrm{p}<0,01$. EACF $F_{50}:$ Extrait Aqueux de C. ferruginea $(50 \mathrm{mg} / \mathrm{kg}$ de P.C) $E_{10 F_{100}}$ : Extrait Aqueux de C. ferruginea $(100 \mathrm{mg} / \mathrm{kg}$ de P.C) 


\section{Effets de Cnestis ferruginea sur le poids de l'ovaire, l'utérus, du fotus et le placenta}

Le traitement des rattes avant l'accouplement, pendant l'accouplement et au début de la gestation avec l'EACF ne provoque aucune variation significative $(\mathrm{p}>0,05)$ du poids des ovaires comparé aux témoins au $15^{\text {ème }}$ jour de gestation.

Au niveau de l'utérus gestant entier, l'extrait induit une augmentation significative de son poids chez les rattes traitées avec les deux doses. En effet, il a été observé une augmentation de 36,19\% (p<0,05) et de 42,69\% (p<0,001) respectivement avec l'EACF 50 et $\mathrm{l}^{\prime} \mathrm{EACF}_{100}$ comparé aux témoins.

Le poids des fœtus issus des femelles prétraitées a également connu une augmentation significative comparée aux témoins. Ainsi, le poids moyen des fœtus issus des rattes traitées avec l'EACF $\mathrm{A}_{50}$ 'est accru de 17,74\% $(\mathrm{p}<0,01)$ et ceux issus des rattes traitées avec l'EACF 100 a connu une croissance de $30,11 \%(\mathrm{p}<0,001)$.

Les résultats ont montré aussi une augmentation hautement significative $(\mathrm{p}<0,001)$ du poids moyen des placentas des fœtus des rattes prétraitées avec les deux doses. Cette augmentation de poids s'élève à des taux de 26,64\% et de $30,41 \%$ respectivement pour 1 'EACF50 et l'EACF $_{100}$ par rapport aux témoins (Tableau III).

Tableau III : Effets de Cnestis ferruginea sur le poids des ovaires, l'utérus, le foetus et le placenta des rattes traitées pendant l'accouplement et en début de la gestation.

\begin{tabular}{|c|c|c|c|}
\hline Groupe & $\begin{array}{l}\text { Ovaire } \\
(\mathrm{mg} / 100 \mathrm{~g})\end{array}$ & Utérus (mg/100g) & $\begin{array}{l}\text { Foetus } \\
\text { (g) }\end{array}$ \\
\hline
\end{tabular}

Témoin $\quad 0,034 \pm 0,004$

$2,319 \pm 0,217$

$0,186 \pm 0,004$

$0,148 \pm 0,003$

$\mathrm{EACF}_{50}$

$0,037 \pm 0,004$

$3,158 \pm 0,108^{*}$

$0,219 \pm 0,008^{* * *}$

$0,183 \pm 0,005^{* * * *}$

$\mathbf{E A C F}_{100} \quad 0,033 \pm 0,002$

$3,309 \pm 0,1505^{* *}$

$0,242 \pm 0,006^{* * *}$

$0,193 \pm 0,005^{* * *}$

Les valeurs sont des moyennes \pm ESM (n=12/lots). ${ }^{*} \mathrm{p}<0.05, * * \mathrm{p}<0,01, * * * \mathrm{p}<0,001$.

$E A C F_{50}$ : Extrait Aqueux de C. ferruginea $(50 \mathrm{mg} / \mathrm{kg}$ de P.C)

EACF $_{100}$ : Extrait Aqueux de C. ferruginea $(100 \mathrm{mg} / \mathrm{kg}$ de P.C)

Effets de Cnestis ferruginea sur la fertilité du rat

Effets sur les paramètres de la fertilité des rats mâles

L'administration par voie orale d'EACF 50 et d'EACF 100 à des rats mâles et accouplés au $50^{\text {ème }}$ jour de traitement avec des femelles qui ont prouvées leur fertilité et traitées pendant l'accouplement jusqu'au $60^{\text {ème }}$ jour a permis 
d'apprécier le degré de fertilisation de l'extrait de plante. Ainsi, à la fin du traitement au $60^{\text {ème }}$ jour ( $10^{\text {ème }}$ jour d'accouplement), il a été enregistré $83,33 \%$ et $100 \%$ de rattes gestantes respectivement pour les doses de $\mathrm{l}^{\prime} \mathrm{EACF}_{50}$ et de $l^{\prime} \mathrm{EACF}_{100}$ par rapport au témoin $(75 \%)$. Les résultats ont également montré que le pourcentage de rats ayant engendré une gestation au cours des 10 jours de traitement était de 33,33\% et ceux ayant engendré deux gestations était de $66,67 \%$ pour l'EACF 50 . Pour l'EACF $100,100 \%$ des rats traités avaient engendré deux gestations (Tableau IV).

En effet, les résultats ont montré que pour chacune des deux doses et les témoins, l'index de copulation et l'index de fertilité étaient identiques dans chaque cas. Ainsi, les index de copulation et de fertilité étaient de 75\%, $83,33 \%$ et $100 \%$ respectivement pour les témoins, traités $\mathrm{EACF}_{50}$ et traités $\mathrm{EACF}_{100}$. A l'instar des témoins, les lots traités ont présenté un taux d'index de fécondité de $100 \%$. (Tableau V). 
Tableau IV : Effets de l'extrait aqueux des feuilles de Cnestis ferruginea sur la fertilisation du rat mâle

\begin{tabular}{ccccccc}
\hline Groupe & Nb de mâle & Nb de femelle & $\begin{array}{c}\text { Nb de femelle } \\
\text { gestante }\end{array}$ & $\begin{array}{c}\text { \% de mâle } \\
\text { n'ayant } \\
\text { engendré aucune } \\
\text { gestation }\end{array}$ & $\begin{array}{c}\text { \% de mâle ayant } \\
\text { engendré une } \\
\text { gestation }\end{array}$ & $\begin{array}{c}\text { \% de mâle ayant } \\
\text { engendré deux } \\
\text { gestations }\end{array}$ \\
\hline Témoin & 6 & 12 & 0 & 0 & 50 & 50 \\
EACF $_{50}$ & 6 & 12 & 10 & 0 & 33,33 & 66,67 \\
EACF $_{\mathbf{1 0 0}}$ & 6 & 12 & 12 & 0 & 0 & 100 \\
\hline
\end{tabular}

Les valeurs sont des moyennes \pm ESM (n=12/lots).

$E_{\text {ECF }}$ : Extrait Aqueux de C. ferruginea $(50 \mathrm{mg} / \mathrm{kg}$ de P.C)

$E A C F_{100}$ : Extrait Aqueux de C. ferruginea $(100 \mathrm{mg} / \mathrm{kg}$ de P.C)

Tableau V : Effets de l'extrait aqueux des feuilles de Cnestis ferruginea sur les index de copulation, de fécondité et de fertilité du rat.

\begin{tabular}{cccc}
\hline Groupe & Index de copulation $(\%)$ & Index de fécondité $(\%)$ & Index fertilité $(\%)$ \\
\hline Témoin & 75 & 100 & 75 \\
EACF $_{\mathbf{5 0}}$ & 83,33 & 100 & 83,33 \\
EACF $_{\mathbf{1 0 0}}$ & 100 & 100 & 100 \\
\hline
\end{tabular}

Les valeurs sont des moyennes \pm ESM (n=12/lots).

$E A C F_{50}$ : Extrait Aqueux de C. ferruginea $(50 \mathrm{mg} / \mathrm{kg}$ de P.C)

$E A C F_{100}$ : Extrait Aqueux de C. ferruginea (100 mg/kg de P.C) 


\section{Effets de Cnestis ferruginea sur la fertilité des rats mâles et femelles} Effets sur quelques paramètres de la fertilité et le taux de gestation en fonction du temps

Les rats et les rattes en cours de traitement $(60$ et 30 jours respectivement), placées en en cage d'accouplement (au 50ème jour de traitement pour les mâles et au 20 ème jour pour les femelles) ont présenté un taux de gestation identique quelle que soit la dose administrée. En effet, toutes les rattes $(100 \%)$ étaient gestantes dès le deuxième jour d'accouplement. De même, tous les rats mâles $(100 \%)$ ont engendré deux gestations pendant les deux premiers jours d'accouplement quelle que soit la dose. Pendant que les témoins recevant l'eau distillée ont présenté $83,33 \%$ de femelles gestantes. Concernant les mâles témoins, 33,33\% ont engendré une gestation et $66,67 \%$ ont induit deux gestations (Tableau VIII).

Les index de copulation, de fécondité et de fertilité sont identiques quelle que soit la dose administrée (100\%). Avec les témoins le taux d'index de copulation et de fertilité est de $83,33 \%$ dans chaque cas et celui de la fécondité est de $100 \%$. (Tableau IX). 
Tableau VIII : Effets de l'extrait aqueux des feuilles de Cnestis ferruginea quelques paramètres de la fertilité

\begin{tabular}{ccccccc}
\hline Groupe & Nb de mâle & Nb de femelle & $\begin{array}{c}\text { Nb de femelle } \\
\text { gestante }\end{array}$ & $\begin{array}{c}\text { \% de mâle } \\
\text { n'ayant } \\
\text { engendré aucune } \\
\text { gestation }\end{array}$ & $\begin{array}{c}\text { \% de mâle ayant } \\
\text { engendré une } \\
\text { gestation }\end{array}$ & $\begin{array}{c}\text { \% de mâle ayant } \\
\text { engendré deux } \\
\text { gestations }\end{array}$ \\
\hline Témoin & 6 & 12 & 10 & 0 & 33,33 & 66,67 \\
EACF $_{\mathbf{5 0}}$ & 6 & 12 & 12 & 0 & 0 & 100 \\
EACF $_{\mathbf{1 0 0}}$ & 6 & 12 & 12 & 0 & 0 & 100 \\
\hline
\end{tabular}

Les valeurs sont des moyennes \pm ESM (n=12/lots). $* \mathrm{p}<0.05, * * * \mathrm{p}<0,001$.

$E A C F_{50}$ : Extrait Aqueux de C. ferruginea $(50 \mathrm{mg} / \mathrm{kg}$ de P.C)

$E_{10 F_{100}}$ : Extrait Aqueux de C. ferruginea $(100 \mathrm{mg} / \mathrm{kg}$ de P.C)

Tableau IX : Effets de l'extrait aqueux des feuilles de Cnestis ferruginea sur les index de copulation, de fécondité et de fertilité du rat.

\begin{tabular}{cccc}
\hline Groupe & Index de copulation $(\%)$ & Index de fécondité $(\%)$ & Index fertilité $(\%)$ \\
\hline Témoin & 83,33 & 100 & 83,33 \\
EACF $_{\mathbf{5 0}}$ & 100 & 100 & 100 \\
EACF $_{\mathbf{1 0 0}}$ & 100 & 100 & 100 \\
\hline
\end{tabular}

Les valeurs sont des moyennes \pm ESM ( $\mathrm{n}=12 /$ lots). ${ }^{*} \mathrm{p}<0.05,{ }^{* * *} \mathrm{p}<0,001$.

$E A C F_{50}$ : Extrait Aqueux de C. ferruginea $(50 \mathrm{mg} / \mathrm{kg}$ de P.C)

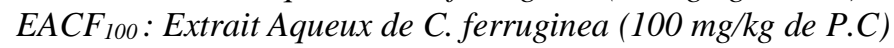




\section{Effets de Cnestis ferruginea sur le poids corporel des rattes gestantes}

Les rattes ont présenté un poids corporel moyen presque identique au premier jour de gestation quelle que soit la dose administrée. Au 15ème jour de gestation, les résultats ont montré une croissance significative $(\mathrm{p}<0,05)$ de $11,79 \%$ du poids corporel des rattes recevant 1'EACF50 par rapport aux témoins (Tableau X). Alors qu'il est observé une diminution non significative du poids corporel des rattes recevant la forte dose d'extrait (EACF100).

\section{Effets de Cnestis ferruginea sur quelques paramètres de la fertilité après autopsie}

Les rattes et les rats traités avec l'EACF et accouplée pendant 10 jours (pendant lesquels les animaux ont continué à être traité) ont montré une augmentation significative $(\mathrm{p}<0,05)$ du nombre de site d'implantation quelle que soit la dose. En effet, le nombre de site d'implantation a connu un taux d'augmentation de $18,95 \%$ et de $31,20 \%$ respectivement avec 1'EACF50 et l'EACF100 comparé aux témoins. Ainsi, le pourcentage d'avortement préimplantation a connu une baisse suite au traitement. Ces taux sont de 20,46\% et $17,65 \%$ respectivement avec l'EACF50 et l'EACF100; alors que ce taux est de $27,93 \%$ pour les femelles recevant de l'eau distillée (témoin) accouplées avec les mâles recevant le même traitement.

Le dénombrement des sites de résorption chez ces rattes traitées avec l'EACF100 et accouplées avec des mâles traités avec la même dose a montré une augmentation hautement significative $(\mathrm{p}<0,001)$ de $9,98 \%$ par rapport au témoin. Avec 1'EACF50, il n'a été observé aucun site de résorption chez ces rattes. Ainsi, le taux d'avortement post-implantation est nul chez les sujets recevant l'EACF50 pendant que ce taux s'élève à $17,76 \%$ chez les rattes traitées avec l'EACF100 et accouplées avec les mâles recevant la même dose. Chez les témoins ce taux est de $3,17 \%$.

Dans le cas du nombre de fœtus viable, les résultats ont montré une augmentation significative $(\mathrm{p}<0,05)$ de 22,85\% avec l'EACF50. La différence observée avec l'EACF100 n'est pas significative (Tableau XI). 
Tableau X : Effets de Cnestis ferruginea sur le poids corporel des rattes gestantes

\begin{tabular}{|c|c|c|}
\hline Groupe & $\begin{array}{c}\text { Poids } \\
\text { Initial (g) }\end{array}$ & $\begin{array}{c}\text { Poids } \\
\text { Final (g) }\end{array}$ \\
\hline Témoin & $145,100 \pm 3,345$ & $183,200 \pm 2,958$ \\
\hline $\mathbf{E A C F}_{50}$ & $145,300 \pm 2,712$ & $204,800 \pm 4,886^{*}$ \\
\hline $\mathrm{EACF}_{100}$ & $145,700 \pm 2,368$ & $187,100 \pm 5,179$ \\
\hline
\end{tabular}

Tableau XI : Effets de l'extrait aqueux des feuilles de Cnestis ferruginea sur quelques paramètres de la fertilité et le poids du fotus et le placenta

\begin{tabular}{cccccc}
\hline Groupe & Nb de corps jaunes & $\begin{array}{c}\text { Nb de site } \\
\text { d'implantation }\end{array}$ & $\begin{array}{c}\text { Nb de site de } \\
\text { résorption }\end{array}$ & $\begin{array}{c}\text { Nb de foetus viable } \\
\text { Avortement pré- } \\
\text { implantation (\%) }\end{array}$ & $\begin{array}{c}\text { Avortement } \\
\text { post- } \\
\text { implantation } \\
(\%)\end{array}$ \\
\hline Témoin & $10,930 \pm 0,742$ & $7,877 \pm 0,654$ & $0,167 \pm 0,112$ & $7,627 \pm 0,507$ & 27,93 \\
EACF $_{\mathbf{5 0}}$ & $11,780 \pm 0,8574$ & $9,370 \pm 0,321^{*}$ & $0,000 \pm 0,000$ & $9,370 \pm 0,321^{*}$ & 20,46 \\
EACF $_{\mathbf{1 0 0}}$ & $12,550 \pm 0,678$ & $10,335 \pm 0,476^{*}$ & $1,833 \pm 405^{* * *}$ & 8,17 \\
\hline
\end{tabular}

Les valeurs sont des moyennes \pm ESM (n=12/lots). *p<0.05, *** $\mathrm{p}<0,001$.

$E A C F_{50}$ : Extrait Aqueux de C. ferruginea $(50 \mathrm{mg} / \mathrm{kg}$ de P.C)

EACF 100 : Extrait Aqueux de C. ferruginea (100 mg/kg de P.C) 


\section{Effets de Cnestis ferruginea sur le poids de l'ovaire, de l'utérus, du fotus et du placenta}

Les résultats ont montré que l'extrait n'induit pas de variation significative $(\mathrm{p}>0,05)$ du poids des ovaires des rattes traitées et accouplées avec les mâles traités également aux différentes doses de l'EACF. Cependant, le poids de l'utérus des rattes traitées avec l'EACF $\mathrm{F}_{50}$ et accouplées avec des mâles traités aussi avec la même dose, augmente très significativement $(\mathrm{p}<0,01)$ de 45,79\% par rapport aux témoins. L'EACF 100 n'a induit aucun changement significatif comparé aux témoins.

Concernant les fœtus des rattes traitées, leur poids a augmenté significativement par rapport aux témoins. Ainsi, les fotus issus du traitement de 1 'EACF 50 se sont accrus de $15,52 \%(\mathrm{p}<0,01)$ et ceux issus du traitement de $1^{\prime} E A C F_{100}$ ont augmenté de $24,71 \%(\mathrm{p}<0,001)$. Le placenta de ces fœtus a également connu une augmentation significative de leur poids par rapport aux témoins. En effet, l'EACF 50 a induit une augmentation de 24,30\% (p<0,01) du poids des placentas. Quant à l'EACF 100 le taux d'augmentation qu'il a induit est de 22,92\% (p<0,05) (Tableau XII).

Tableau XII : Effets de Cnestis ferruginea sur le poids des ovaires, l'utérus, le foetus et le placenta des rattes traitées pendant l'accouplement et début de la gestation

\begin{tabular}{ccccc}
\hline Groupe & $\begin{array}{c}\text { Ovaire } \\
(\mathbf{m g} / \mathbf{1 0 0} \mathbf{g})\end{array}$ & $\begin{array}{c}\text { Utérus } \\
(\mathbf{m g} / \mathbf{1 0 0 g})\end{array}$ & $\begin{array}{c}\text { Fœtus } \\
(\mathbf{g})\end{array}$ & $\begin{array}{c}\text { Placenta } \\
(\mathbf{g})\end{array}$ \\
\hline Témoin & $0,031 \pm 0,002$ & $2,693 \pm 0,08761$ & $0,174 \pm 0,003$ & $0,144 \pm 0,003$ \\
EACF $_{\mathbf{5 0}}$ & $0,035 \pm 0,003$ & $3,926 \pm 0,1885^{* *}$ & $0,201 \pm 0,006^{* *}$ & $0,179 \pm 0,006^{* *}$ \\
EACF $_{\mathbf{1 0 0}}$ & $0,028 \pm 0,002$ & $2,026 \pm 0,332$ & $0,217 \pm 0,009^{* * *}$ & $0,177 \pm 0,013^{*}$ \\
\hline
\end{tabular}

Les valeurs sont des moyennes \pm ESM (n $=12 /$ lots). ${ }^{*} \mathrm{p}<0.05, * * \mathrm{p}<0,01, * * * \mathrm{p}<0,001$.

$E A C F_{50}$ : Extrait Aqueux de C. ferruginea $(50 \mathrm{mg} / \mathrm{kg}$ de P.C)

$E_{C A C} F_{100}$ : Extrait Aqueux de C. ferruginea $(100 \mathrm{mg} / \mathrm{kg}$ de P.C)

\section{Effets de Cnestis ferruginea sur l'histologie de l'ovaire et l'utérus des rattes gestantes \\ Effets sur l'ovaire}

Dans cette étude, l'intérêt a été accordé à l'analyse toxicologique et morphométrique des corps jaunes. En effet, quelle que soit la dose, les rattes ont présenté une architecture normale et donc aucune nécrose ou dégénérescence n'a été constatée dans les corps jaunes (Figure 2).

Les études morphométriques réalisées ont montré que les rattes traitées avec la dose de l'EACF 50 ont connu une augmentation significative $(\mathrm{p}<0,05)$ du diamètre de leurs corps jaunes par rapport aux témoins. Ainsi, le diamètre

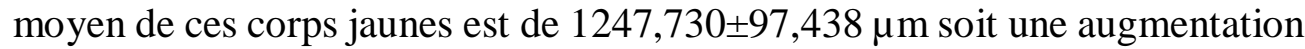
de $35,14 \%$ par rapport aux témoins $(923,310 \pm 89,732 \mu \mathrm{m})$. Le diamètre de corps jaune obtenu avec l'EACF $100 \quad(909,148 \pm 54,526 \mu \mathrm{m})$ n'est pas significativement différent de celui des témoins $(\mathrm{p}>0,05)$. Il faut noter que 
$37,50 \%$ (3/8) des rattes qui recevaient la dose de $100 \mathrm{mg} / \mathrm{kg}$ de PC ont présenté des corps jaunes en régression.
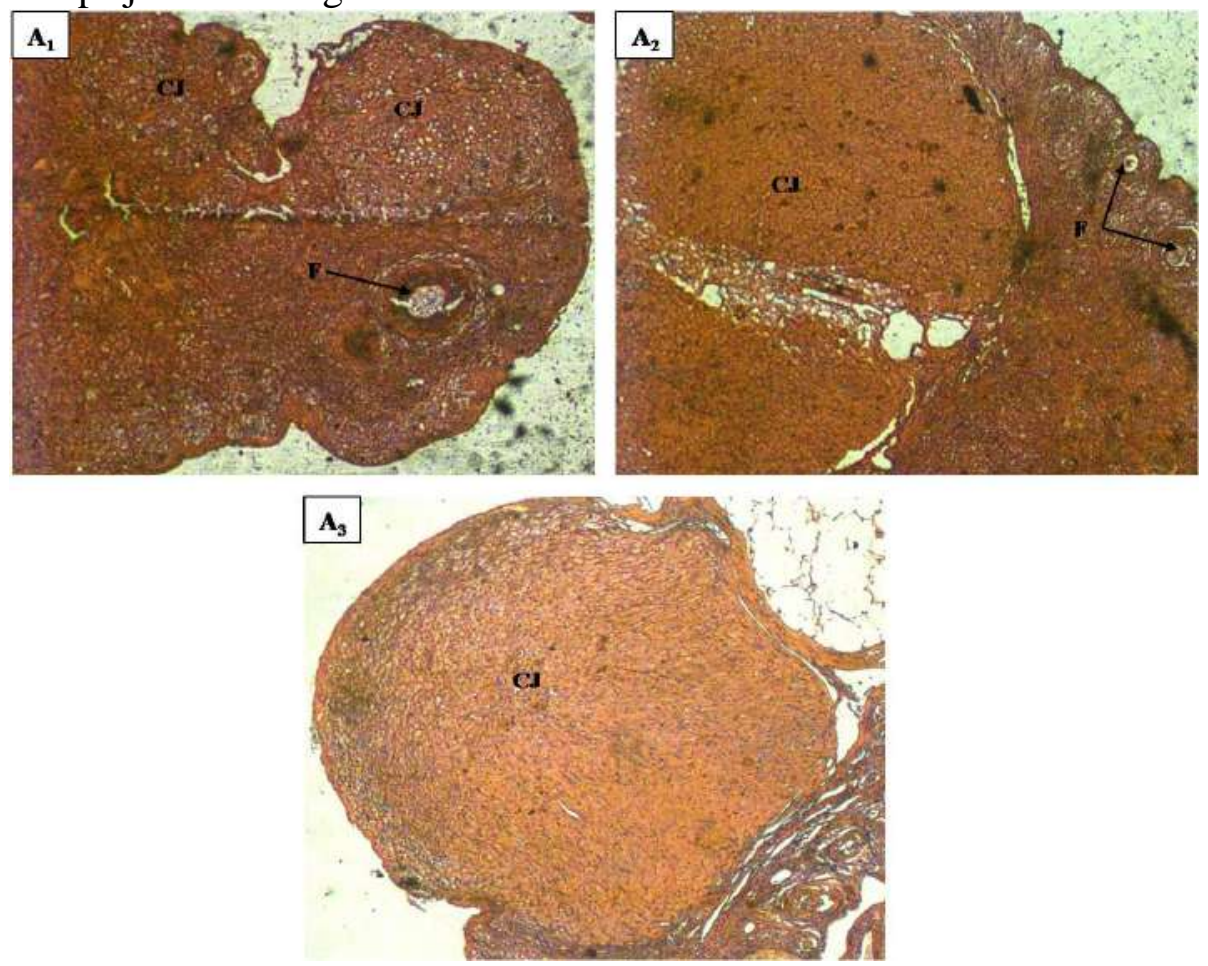

Figure 2 : Micrographie de l'ovaire de rattes gestantes témoins et rattes gestantes traitées avec les doses de Cnestis ferruginea.

$\mathbf{A}_{\mathbf{1}}$ : Témoin ; $\mathbf{A}_{\mathbf{2}}: 50 \mathrm{mg} / \mathrm{kg}$ de PC d'extrait aqueux de Cnestis ferruginea; $\mathbf{A}_{\mathbf{3}}$ : Traité 100 $\mathrm{mg} / \mathrm{kg}$ de PC d'extrait aqueux de Cnestis ferruginea

CJ : Corps jaune $; \mathbf{F}$ : Follicules

Grossissement : $\times 40$; Coloration : Hématoxyline éosine

\section{Effets sur l'utérus}

L'examen histologique réalisé sur les cornes utérines des rattes au 15 ème jour de gestation chez les témoins comme les traitées n'a présenté aucune inflammation ni nécrose cellulaire (Figure 3). Tout comme les témoins, les traitées ont présenté un développement normal des cellules épithéliales de l'endomètre. Cependant, l'étude morphométrique réalisée sur les glandes utérines a montré une augmentation significative $(\mathrm{p}<0,05)$ du diamètre de celles-ci chez les rattes traitées avec l'EACF50 et l'EACF100 (Tableau XIII). Cette augmentation était respectivement de 16,05\% et de 18,39\% par rapport aux témoins.Concernant le diamètre des vaisseaux sanguins, le traitement n'a induit aucune variation significative de ceux-ci. 
Figure 2 : Micrographie de l'utérus de rattes gestantes témoins et rattes gestantes traitées avec les doses de Cnestis ferruginea.
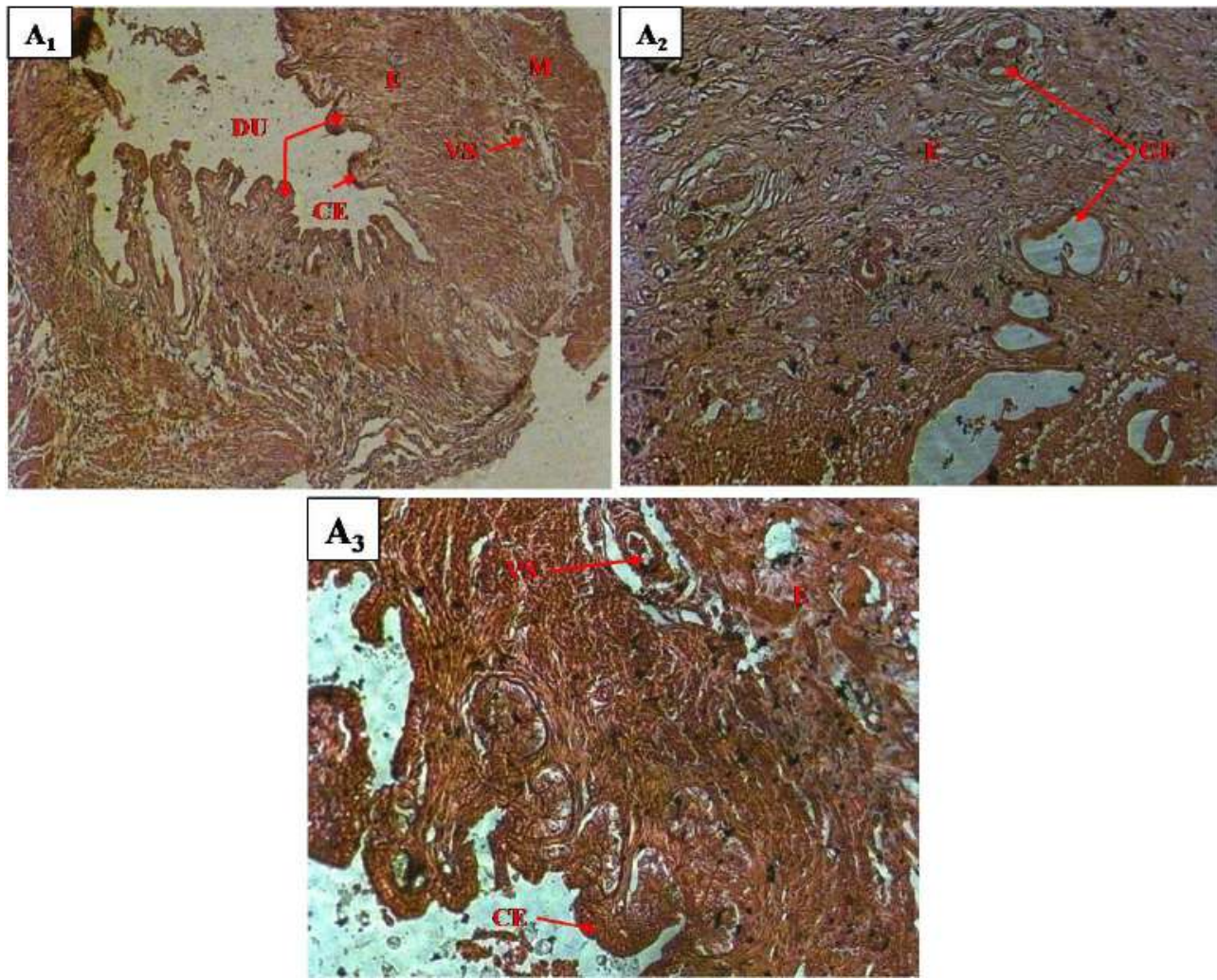

$\mathbf{A}_{\mathbf{1}}$ : Témoin ; $\mathbf{A}_{\mathbf{2}}: 50 \mathrm{mg} / \mathrm{kg}$ de PC d'extrait aqueux de Cnestis ferruginea; $\mathbf{A}_{\mathbf{3}}$ : Traité 100 $\mathrm{mg} / \mathrm{kg}$ de PC d'extrait aqueux de Cnestis ferruginea

$\mathbf{C E}$ : Cellules épithéliales ; DU : Dentelle utérine ; $\mathbf{E}$ : Endomètre ; GU : Glande utérine ; $\mathbf{M}$ : Myomètre; VS : Vaisseau sanguin.

Grossissement : $\times 100$; Coloration : Hématoxyline éosine

Tableau XIII : Effets de Cnestis ferrugiea sur les diamètres des glandes utérines et des vaisseaux sanguins des rattes gestantes.

\begin{tabular}{cccc}
\hline Paramètres & Témoin & EACF $_{\mathbf{5 0}}$ & EACF $_{\mathbf{1 0 0}}$ \\
\hline $\begin{array}{c}\text { Diamètres des } \\
\text { glandes utérines } \\
\text { Diamètres des } \\
\text { vaisseaux } \\
\text { sanguins }\end{array}$ & $67.120 \pm 9.839$ & $77.895 \pm 11.147^{*}$ & $79,463 \pm 8.732^{*}$ \\
\hline
\end{tabular}

Les valeurs sont des moyennes \pm ESM (n=12/lots). *p $<0.05$, **p $<0,01, * * * \mathrm{p}<0,001$.

EACF50: Extrait Aqueux de C. ferruginea $(50 \mathrm{mg} / \mathrm{kg}$ de P.C)

EACF 100 : Extrait Aqueux de C. ferruginea $(100 \mathrm{mg} / \mathrm{kg}$ de P.C)

\section{Discussion}

Les rattes en cours de traitement de 30 jours, placées en présence de

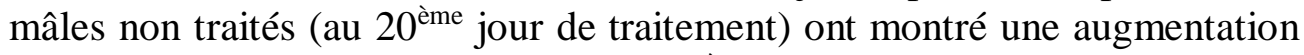
significative de leur poids corporel au $15^{\mathrm{ème}}$ jour de gestation par rapport au 
témoin. Ce résultat pourrait s'expliquer par la valeur nutritionnelle de cette plante (Irvine, 1961). L'extrait pourrait avoir également un effet stimulateur sur l'appétit et ainsi augmenter la prise alimentaire. Il est évident que l'état de gestation entraîne une prise de poids chez la femelle. Cette augmentation de poids pourrait être liée au nombre de foetus porté par la femelle.

Concernant le délai de copulation, les résultats montrent que dès le $3^{\text {ème }}$ jour de traitement, toutes les rattes traitées étaient gestantes avec un index de copulation, de fécondité et de fertilité qui étaient de $100 \%$. Ces données sont en conformité avec les effets induits par l'extrait sur le cycle œstral des rattes (Zougrou et al., 2016). En effet, dans cette étude, l'extrait aqueux de $C$. ferruginea a induit un blocage du cycle œstral des femelles traitées en œstrus rendant ainsi ces rattes permanemment réceptives. En plus, l'extrait induisait l'ovulation (Zougrou et al., 2018a). Cette dernière hypothèse est renforcée par l'augmentation du nombre de corps jaune enregistrée dans l'étude présente. La différence du nombre de corps jaune, quoique non significative, renseigne sur l'ovulation induite par l'extrait. De même, l'extrait pourrait favoriser le maintien de la phase lutéinique pendant le début de la gestation.

Par ailleurs, l'augmentation du nombre de sites d'implantation, corrélée par la baisse du taux d'avortement pré-implantation pourrait être imputée à l'extrait. En effet, le développement régulier de tous les évènements menant à la nidation, au moins chez le rat et la souris, est principalement sous la commande directe de l'effet œstrogène-progestérone au niveau cellulaire (Psychoyos et Prapas, 1987). Ainsi, l'extrait agirait favorablement dans l'établissement de l'équilibre de ces deux hormones afin de faciliter la nidation. Ces résultats sont différents de l'administration de vorinostat aux rattes Sprague-Dawley (Wise et al., 2008). En effet, l'administration de cette substance induit une augmentation du pourcentage d'avortement préimplantation.

Au niveau des sites de résorption et du pourcentage d'avortement postimplantation les résultats ont montré une baisse de ces paramètres avec la dose de $50 \mathrm{mg} / \mathrm{kg}$ de PC (l'EACF 50$)$. Ceci est la preuve d'une très bonne imprégnation hormonale de l'endomètre des rattes traitées pour une bonne nidation des fœtus et à les garder jusqu'à terme. Inversement, la dose de 100 $\mathrm{mg} / \mathrm{kg}$ de $\mathrm{PC}\left(\mathrm{EACF}_{100}\right)$ a présenté un nombre significatif de site de résorption et un taux élevé de pourcentage post-implantation par rapport au témoin. Ce résultat pourrait être lié à la dose utilisée. Ainsi, cette forte dose pourrait être à l'origine d'une perturbation de l'équilibre endocrinien requis pour maintenir la gestation. Cela pourrait également être dû à une toxicité directe de l'extrait sur le fœtus. En effet, la toxicité fotale est principalement exprimée par la réduction du poids corporel et une augmentation du nombre de site de résorptions. Cependant, en embryo-toxicité, il est nécessaire de séparer la toxicité directe sur le foetus des effets secondaires dérivés de la toxicité 
maternelle (Khera, 1984). Dans cette étude, il est plausible que cette toxicité se manifeste directement sur l'embryon.

Malgré cette toxicité constatée avec la forte dose, le nombre de fœus viable a significativement augmenté pour les deux doses utilisées dans cette expérience. Ce résultat vient expliquer le fait que la toxicité exercée à la dose de $100 \mathrm{mg} / \mathrm{kg}$ de PC serait faible. Cette toxicité serait due à la composition de l'extrait. En effet, les constituants tels que les alcaloïdes, les flavonoïdes et les saponosides qui sont présents dans l'extrait de $C$. ferruginea (Yakubu et al., 2011 ; Zougrou, 2017) sont reconnus pour leurs activités contraceptives et antifertilisantes. Ainsi, ces constituants pourraient agir individuellement ou en synergie pour affecter l'utérus et mener à l'avortement.

En ce qui concerne le poids de l'utérus gestant, il a été enregistré une augmentation significative de son poids par rapport au témoin. Cette différence de poids pourrait s'expliquer par le nombre de fœtus contenu dans les cornes de l'utérus. Il a été observé également une augmentation du poids des fotus et des placentas issus des rattes ayant reçues l'extrait de $C$. ferruginea. Ce résultat serait également lié à la valeur nutritionnelle évoquée plus haut de cet extrait de plante.

Par ailleurs, l'augmentation de poids des fotus et des placentas est dose dépendante. En effet, ce résultat pourrait suggérer que l'extrait est à la base de l'augmentation des sites de résorptions et donc du pourcentage d'avortement post-implantation. Ainsi, un grand nombre de fœetus dans l'utérus avec une croissance rapide pourrait créer un encombrement qui serait à l'origine de la perte des embryons.

Les paramètres spermatiques que sont la densité, la motilité et la morphologie sont considérés comme la clé de la fertilité chez le mâle. Dans l'étude menée par Zougrou et al., (2018b), les mâles traités avec l'extrait aqueux de $C$. ferruginea ont montré une augmentation significative des taux de spermatozoïdes mobiles, de leurs nombres et des spermatozoïdes normaux. Dans ce présent test de fertilité, les rats mâles traités avec la dose de $50 \mathrm{mg} / \mathrm{kg}$ de PC et croisés avec les femelles non traitées, ont tous engendré une gestation dont $66,67 \%$ ont occasionné 2 gestations. Avec la plus forte dose (EACF 100$)$ tous les rats traités $(100 \%)$ ont engendré 2 gestations. Par ailleurs, il a été montré que l'extrait aqueux des racines de $C$. ferruginea a la capacité de restaurer la compétence sexuelle au moins à un niveau raisonnable chez les rats qui ont subi une induction de dysfonctionnement sexuel par l'administration de $10 \mathrm{mg} / \mathrm{kg}$ de paroxetine (Yakubu et Nurudeen, 2012). Ces auteurs ont également expliqué que la stimulation de cette activité sexuelle qui peut se faire via un mécanisme central et périphérique serait due à la présence de molécules phytochimiques telles que les saponosides, flavonoïdes et les alcaloïdes. Il faut noter que ces composés se sont révélés aussi présents dans 
les feuilles de cette plante (Zougrou, 2017). L'extrait des feuilles pourrait également agir selon le même procédé pour stimuler la libido des rats traités.

En plus, ces rats traités ont montré un index de fécondité et de fertilité beaucoup plus élevé que les témoins de façon dose dépendante. Ce test a également montré une augmentation significative du nombre de sites d'implantation et du nombre de fœtus viable. Ces résultats sont le reflet des effets de l'extrait de plante sur les paramètres spermatiques.

Le test de fertilité réalisé chez les rats mâles traités et les femelles traitées a été effectué pour lever la dernière équivoque sur la fertilisation de l'extrait de $C$. ferruginea. En effet, le taux de gestation, le nombre de mâle ayant engendré deux gestations, l'index de copulation, l'index de fécondité et l'index de fertilité dans cette partie de l'étude viennent une fois encore confirmer les résultats précédents (induction de l'œstrus, et de l'ovulation chez la femelle, augmentation des paramètres spermatiques chez le mâle). Comparativement aux résultats du test de femelles traitées croisées avec les mâles non traités et les mâles traités croisés avec les femelles non traitées, les variations dans certains résultats seraient dues au fait que l'extrait de plante a stimulé le désir sexuel chez les mâles et induit l'œstrus chez la femelle (Yakubu et Nurudeen, 2012 ; Zougrou et al., 2016).

Tout comme dans le test de femelles traitées croisées avec les mâles non traités, l'extrait a induit une augmentation significative du poids corporel des rattes gestantes avec la dose de $50 \mathrm{mg} / \mathrm{kg}$ de PC. Par ce résultat, l'extrait serait non toxique pour les rattes gestantes.

Concernant les paramètres enregistrés au cours de l'autopsie, les variations corroborent les résultats des autres tests de fertilité. En effet, dans ce présent test le taux d'avortement pré- et post-implantation est beaucoup plus important comparé aux témoins du même test et aussi plus important que ceux des tests précédents avec la dose de $100 \mathrm{mg} / \mathrm{kg}$ de PC ; ceci conforte l'idée selon laquelle l'extrait serait abortif à forte dose.

Les résultats sur le poids de l'ovaire, l'utérus, les fœtus et le placenta concordent également avec ceux obtenus au cours du test de fertilité réalisé avec les femelles traitées. Cependant, la dose de $100 \mathrm{mg} / \mathrm{kg}$ de PC n’a pu induire une augmentation significative du poids de l'utérus. Ce résultat pourrait être lié au fort taux d'avortement pré- et post-implantation.

L'étude histologique réalisée sur l'ovaire des rattes traitées avant et en début de gestation a montré une augmentation significative du diamètre des corps jaunes avec la dose de $50 \mathrm{mg} / \mathrm{kg}$ de PC. Ceci suggère que le corps jaune était maintenu en fonction chez ces rattes. En effet, il a été démontré que le maintien du corps jaune est assuré par la prostaglandine E (PGE) sécrété par l'utérus (Gayrard, 2007). Ainsi, la persistance et la grande taille du corps jaune observées dans cette étude seraient liées à une stimulation de la PGE utérine par l'extrait de plante et/ou l'extrait pourrait se comporter comme un agoniste 
de cette substance lutéotrophique et mimer son activité. Le maintien fonctionnel du corps jaune pourrait être également un résultat de la suppression de la sécrétion de PGF2 $\alpha$. En effet, selon Gayrard (2007), cette substance a été identifiée comme la lutéolysine sécrétée par l'endomètre. Ainsi, l'extrait de Cnestis ferruginea pourrait bloquer la libération de la PGF2 $\alpha$.

Ce bon fonctionnement du corps jaune pourrait expliquer aussi la baisse du taux d'avortement constatée dans les études précédentes avec cette même dose $\left(\mathrm{EACF}_{50}\right)$.

Avec la dose de $100 \mathrm{mg} / \mathrm{kg}$ de $\mathrm{PC}$, le diamètre moyen des corps jaunes quoi que non significatif, est inférieur à ceux des témoins et il a été constaté une régression des corps jaunes. Ce résultat serait lié à la dose administrée aux rattes.

Par ailleurs, ce résultat pourrait également expliquer le taux élevé d'avortement avec cette dose. En effet, Il a été établi que plusieurs pertes d'embryons, entre 25 et $55 \%$, des mammifères en début de gestation est dues à une inadéquation de la fonction des cellules lutéales (Niswender et Nett, 1994).

En outre, la régression des corps jaunes serait à l'origine d'une baisse de la libération de la progestérone. Selon Vaissaire (1977), cette hormone est prépondérante à la préparation de l'utérus à la nidation. Le rôle de la progestérone s'étend également au maintien de la grossesse en favorisant la fourniture de nutriments au fotus.

Au niveau de l'utérus, les animaux traités avec la dose de 50 et 100 $\mathrm{mg} / \mathrm{kg}$ de PC ont connu une croissance significative du diamètre des glandes utérines comparé aux témoins. En effet, les glandes utérines ont pour fonctions de synthétiser du glycogène qui est une importante source de nutriment pour l'œuf « fécondé ». Ce résultat vient étayer une fois encore les effets de l'extrait de Cnestis ferruginea sur l'augmentation du poids des fotus constaté plus haut.

\section{Conclusion}

L'extrait de $C$. ferruginea améliore la fertilité en augmentant le nombre de femelles gestantes, le nombre de sites d'implantation et le nombre de fœus viables chez les rattes traitées. Chez les mâles traités, l'extrait de cette plante induit une augmentation de l'index de fécondité et de fertilité et provoque également chez les femelles accouplées avec les mâles traités une augmentation du nombre de sites d'implantation. En outre l'extrait entraîne une augmentation du poids des fotus et du placenta. Mais, ces effets positifs peuvent être entravés par les fortes doses qui provoquent une augmentation du taux d'avortement post-implantation. 


\section{References:}

1. Adisa, R.A., Choudhary, M.I., Adewoye, E.O., \& Olorunsogo, O.O. (2010). Hypoglycaemic and biochemical properties of cnestis ferruginea. Afr. J. Trad. CAM, 7(3): 185-194.

2. Akharaiyi, F.C., Boboye, B.E. \& Adetuyi, F.C. (2012). Hepatoprotective effect of ethanol leaf extract of Cnestis ferruginea on Swiss albino mice induced with paracetamol. Int. Res. J. of Pharmaceuticals. 2(4) : 120-126.

3. Berhaut, J. (1954). Flore du Sénégal, brousses et jardin (savanes de l'Afrique occidentale). Librairie Clairafrique, Dakar, 300p

4. Berhaut, J. (1967). Flore du Sénégal. Edition Clairafrique 485 P.

5. Burkill, H.M. (1985). The useful plants of west tropical africa. Royal Botanic Gardens, kew(K). Vol. 1 : 4 P

6. Declume, C., Assamoi, A. \& Akre, T.B. (1984). Anticonvulsant activity of Cnestis ferruginea, DC. (Connaraceae). Ann Pharm Fr., 42(1) : 35-41.

7. Gayrard, V. (2007). Physiologie de la reproduction des mammifères. Ecole nationale vétérinaire, Toulouse, France, 198 p.

8. Gill, L.S. (1992). Ethnomedical uses of plants in Nigeria, Benin, Nigeria: Uniben Press. P.77.

9. Hafez, E.S.E. (1970). Reproduction and breeding techniques for laboratory animals. Philadelphia, Lea and Febiger

10. Irvine, F.R. (1961). Woody plants of Ghana. Oxford University Press, Oxford. Pp. 146-147

11. Ishola, I.O. \& Ashorobi, R.B. (2007). The anti-stress potential of the aqueous root extract of Cnestis ferruginea. J. Pharm. 3(3) : 295-298.

12. Ishola, I.O., Akindele, A.J. \& Adeyemi, O.O. (2011). Analgesic and anti-inflammatory activities of Cnestis ferruginea Vahl ex DC (Connaraceae) methanolic root extract. J. Ethnopharmacol. 135(1) : 55-62.

13. Kerharo, J. \& Adam, J.G. (1974). La pharmacopée sénégalaise traditionnelle : plantes médicinales et toxiques Editions VIGOT frères, paris, $1011 \mathrm{p}$.

14. Kerharo, J. (1974). La pharmacopée Sénégalaise traditionnelle, plantes médicinales et toxique. Vigot, Paris, 1012p.

15. Khera, K.S. (1984). Maternal toxicity - A possible factor in fetal malformation in mice. Teratology. 29: 411-416.

16. N'guessan, K., Kouadio K. \& Kouamé N.F. (2006). Plantes emménagogues utilisées en médecine traditionnelle par les peuples Abbey et Krobou d'Agboville (côte d'ivoire) Parm. Méd. Afr. 14 : 137-158. 
17. Niswender, G.D. \& Nett, T.M. (1994). Corpus luteum and its control in infraprimate species.P781 in The Physiology of Reproduction. $2^{\text {nd }}$ ed. Knobil E and Neill DJ, ed. Raven Press, Ltd., New York.

18. Okafor, J. \& Ham, R. (1999). Identification, utilisation et conservation des plantes médicinales dans le sud-est du Nigéria. Thème Biodiversité Africaine $3: 1-8$.

19. Psychoyos, A. \& Prapas, I. (1987). Inhibition of egg development and implantation in rats after post-coital administration of the progesterone agonist RU 486. J. Reprod. Fertil. 80: 487-91.

20. Vaissaire, J.P. (1977). Sexualité et reproduction des mammifères domestiques et de laboratoire. Paris :Maloine Editeur. 457 P.

21. Wise, L.D., Spence, S., Saldutti, L.P. \& Kerr, J.S. (2008). Assessment of female and male fertility in Sprague-Dawley rats administered vorinostat, a histone deacetylase inhibitor. Birth Defects Research (Part B). 83:19-26.

22. Yakubu, M.T. \& Nurudeen, Q.O. (2012). Effects of aqueous extract of Cnestis ferruginea (Vahl ex De Cantolle) root on paroxetine-induced sexual dysfunction in male rats. Asian Pac. J. Reprod., ;1(2) : 111-116.

23. Yakubu, M.T., Adams, D.M., Akanji, M.A. \& Oladiji, A.T. (2011). Laxative activity of the aqueous root extract of Cnestis ferruginea (Vahl ex DC) in loperamide-induced constipated rats. Nig. J. Gastroenterol \& Hepatol.3(1-2): 21-29

24. Zougrou, N.E., Blahi, A.N., D’Almeida, M.-A. \& Kouakou, K. (2016). Fertility enhancing effects of aqueous extract of leaves of Cnestis ferruginea Vahl ex De Cantolle on female Wistar rats. Int. J. Biosci. 9(6): 79-91.

25. Zougrou, N.E., Blahi, A.N., Kouassi, K.D. \& Kouakou, K. (2018a). Effects of the aqueous extract of Cnestis ferruginea on the histological structure of female rat ovary and uterine horns. Biomed. J. Sci. \& Tech. Res. 2(1) BJSTR. MS.ID.000625. DOI : 10.26717/BJSTR.2018.02.000625.

26. Zougrou, N.E. (2017). Evaluation des effets toxicologiques et pharmacologiques d'un extrait aqueux de Cnestis ferruginea Vahl ex DC. (Connaraceae) sur le systeme reproducteur des rats mâle et femelle. Thèse de doctorat, Université Félix Houphouët-Boigny Abidjan Côte d'Ivoire. 226p.

27. Zougrou, N.E., Kouassi, K.A., Tahiri, A., Blahi, A.N.M. \& Kouakou, K. (2018b). Evaluation of the effects of aqueous leaves extract of Cnestis ferruginea from Côte d'Ivoire on male rat reproductive system. European J. Med. Plants. 25(3): 1-16. DOI: 10.9734/EJMP/2018/43492 